\title{
4 Der Hochschulstandort Wien im Überblick
}

\subsection{Die tertiären Bildungseinrichtungen Wiens in der österreichischen Hochschullandschaft}

Der Wiener Hochschulstandort ist durch zwei Charakteristika geprägt: erstens die Dominanz innerhalb der österreichischen Hochschullandschaft, die sich aus der historischen Tradition der großen Universitäten ergibt. An den drei großen Wiener Uni-

Tabelle 4.1: Hochschulen in Wien (WS 2012) im nationalen Vergleich

\begin{tabular}{lrrr}
\hline \multicolumn{1}{c}{ Name (Gründungsjahr) } & Studierende & \multicolumn{2}{c}{ Anteil der Studierenden (\%) } \\
& gesamt & Österreich & Wien \\
\hline Universität Wien (1365) & 92.485 & 26,6 & 49,5 \\
Medizinische Universität Wien (2004) & 7.468 & 2,2 & 4,0 \\
Technische Universität Wien (1815) & 27.923 & 8,0 & 14,9 \\
Universität für Bodenkultur Wien (1872) & 11.393 & 3,3 & 6,1 \\
Veterinärmedizinische Universität Wien (1765) & 2.286 & 0,7 & 1,2 \\
Wirtschaftsuniversität Wien (1898) & 23.584 & 6,8 & 12,6 \\
Universität für angewandte Kunst Wien (1867) & 1.716 & 0,5 & 0,9 \\
Universität für Musik und darst. Kunst Wien (1819) & 3.243 & 0,9 & 1,7 \\
Akademie der bildenden Künste Wien (1692) & 1.434 & 0,4 & 0,8 \\
\hline Universitäten gesamt & $\mathbf{1 7 1 . 5 3 2}$ & $\mathbf{4 9 , 4}$ & $\mathbf{9 1 , 7}$ \\
\hline Fachhochschule Campus Wien (2001) & 4.022 & 1,2 & 2,2 \\
Fachhochschule des bfi Wien GmbH (1996) & 1.854 & 0,5 & 1,0 \\
Fachhochschule Technikum Wien (1994) & 3.382 & 1,0 & 1,8 \\
Ferdinand Porsche FernFH (1997) & 538 & 0,2 & 0,3 \\
FH Wien der WK Wien (1994) & 2.364 & 0,7 & 1,3 \\
\hline Fachhochschulen gesamt & $\mathbf{1 2 . 1 6 1}$ & $\mathbf{3 , 5}$ & $\mathbf{6 , 5}$ \\
\hline Modul University Vienna (2007) & 235 & 0,1 & 0,1 \\
Privatuniversität Konservatorium Wien (2005) & 922 & 0,3 & 0,5 \\
Sigmund Freud Privatuniversität Wien (2005) & 923 & 0,3 & 0,5 \\
Webster University Wien (2001) & 533 & 0,2 & 0,3 \\
\hline Privatuniversitäten gesamt & $\mathbf{2 . 6 1 3}$ & $\mathbf{0 , 8}$ & $\mathbf{1 , 4}$ \\
\hline Sonstige FHs und Privatuniversitäten & $\mathbf{6 8 6 . 9 9 0}$ & $\mathbf{0 , 2}$ & $\mathbf{0 , 4}$ \\
\hline Wien gesamt & $\mathbf{3 4 7 . 1 0 2}$ & $\mathbf{5 3 , 9}$ & $\mathbf{1 0 0 , 0}$ \\
\hline Österreich gesamt & & & \\
\hline & & & \\
\hline & & & \\
\hline
\end{tabular}

Quelle: uni:data 2013. 
versitäten (Universität Wien, TU Wien und WU Wien) sind rund 144.000 oder 41,5\% aller Studierenden in Österreich inskribiert (Tabelle 4.1); in Summe zählen die tertiären Bildungseinrichtungen in Wien 53,9 \% aller österreichischen Studierenden, womit die Dominanz des Standortes Wien in der Hochschullandschaft deutlich gemacht wird. Zweites Charakteristikum des Hochschulstandortes Wien ist die Heterogenität der tertiären Bildungseinrichtungen: Diese differieren beträchtlich hinsichtlich ihrer Größe, ihres Alters, ihres Bildungsangebots sowie ihrer rechtlichen Stellung. Hier sind vor allem die seit Mitte der 1990er Jahre gegründeten Fachhochschulen und Privatuniversitäten zu nennen: Diese Einrichtungen zählen zusammengenommen zwar nur rund 15.500 Studierende (8,3\%), tragen aber zur Diversität des Standortes sowie zur Verbreiterung des Bildungsangebotes bei. Der beträchtlichen Heterogenität der tertiären Bildungseinrichtungen ist in einer Studie, die der Frage regionaler Wertschöpfungseffekte mit einem umfassenden Zugang nachgeht, Rechnung zu tragen.

Tabelle 4.2: Personal (Kopfzahl) an den Universitäten (WS 2012), Fachhochschulen (Studienjahr 2010/11) und Privatuniversitäten (Studienjahr 2011/12) im nationalen Vergleich

\begin{tabular}{|c|c|c|c|c|}
\hline & & Wien & Österreich & Anteil Wien \\
\hline \multirow{8}{*}{ 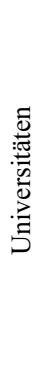 } & Wissenschaftliches Personal gesamt & 17.211 & 35.506 & $48,5 \%$ \\
\hline & Professoren & 1.058 & 2.333 & $45,3 \%$ \\
\hline & wissenschaftliche Mitarbeiter & 16.158 & 33.276 & $48,6 \%$ \\
\hline & Habilitierte & 945 & 2.513 & $37,6 \%$ \\
\hline & assoziierte/Assistenzprofessoren & 133 & 790 & $16,8 \%$ \\
\hline & drittfinanzierte Mitarbeiter & 4.445 & 8.375 & $53,1 \%$ \\
\hline & allgemeines Personal gesamt & 7.389 & 18.028 & $41,0 \%$ \\
\hline & Gesamt & 24.600 & 53.534 & $46,0 \%$ \\
\hline \multirow{7}{*}{ 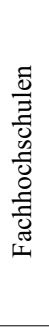 } & Lehrpersonal & 3.872 & 13.186 & $29,4 \%$ \\
\hline & Lehr- und Forschungspersonal & 3.813 & 12.967 & $29,4 \%$ \\
\hline & Studiengangsleiter & 59 & 219 & $26,9 \%$ \\
\hline & Sonstiges Lehrhilfspersonal & 147 & 1.530 & $9,6 \%$ \\
\hline & Akademische Dienste für Studierende & 33 & 174 & $19,0 \%$ \\
\hline & Lehr- und Forschungshilfspersonal & 114 & 1.356 & $8,4 \%$ \\
\hline & Gesamt & 4.019 & 14.716 & $27,3 \%$ \\
\hline \multirow{4}{*}{ 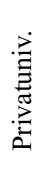 } & Lehre und Forschung & 451 & 1.724 & $26,2 \%$ \\
\hline & Mitarbeit in Lehre und Forschung & 31 & 104 & $29,8 \%$ \\
\hline & Service für Lehre und Forschung & 21 & 57 & $36,8 \%$ \\
\hline & Gesamt & 503 & 1.885 & $26,7 \%$ \\
\hline
\end{tabular}

Quelle: uni:data 2013. Anmerkung: Aufgrund von unterschiedlichen Erhebungszeiträumen weichen diese Daten von den Angaben der Hochschulen in dieser Studie ab. Hier steht die Vergleichbarkeit mit nationalen Daten im Vordergrund. 
Beim Personalstand der Universitäten, Fachhochschulen und Privatuniversitäten bestätigt sich die Dominanz Wiens innerhalb der österreichischen Bildungslandschaft: Knapp die Hälfte des wissenschaftlichen Personals an den Universitäten ist in Wien beschäftigt (48,5 \%, vgl. Tabelle 4.2), wobei es innerhalb der angeführten Untergruppen beträchtliche Abweichungen gibt. Der vergleichsweise geringe Anteil beim allgemeinen Universitätspersonal in Wien (41,0 \%) kann vermutlich auf die Skalen- und Effizienzvorteile zurückgeführt werden, die bei der Verwaltung von großen Einrichtungen lukriert werden können. Insgesamt sind 46,0 \% der Universitätsbeschäftigten an einer Wiener Universität angestellt. Interessant ist, dass im Vergleich dazu bei den Fachhochschulen mit 27,3 \% sowie bei den Privatuniversitäten (26,7 \%) die Dominanz Wiens wesentlich geringer ist. Diese Diskrepanz ist Ausdruck der unterschiedlichen räumlichen Muster eines historisch-zentralisierten Universitätssystems einerseits und dem eines jungen, stark dezentralisierten Fachhochschul-/ Privatuniversitätssystems andererseits. Ein Vergleich der Beschäftigungsformen zwischen den drei Typen ist aufgrund unterschiedlicher Klassifikationen nicht möglich.

Aus dem Verhältnis zwischen Studierendenzahlen und Beschäftigten kann das Betreuungsverhältnis für einzelne Einrichtungen oder Hochschultypen errechnet werden. Um eine Vergleichbarkeit zwischen Universitäten, Fachhochschulen und Privatuniversitäten zu ermöglichen, wurde nicht - wie üblich - das (habilitierte) Lehrpersonal,

Abbildung 4.1: Betreuungsquote (Studierende pro Beschäftigte) an Universitäten, Fachhochschulen und Privatuniversitäten im nationalen Vergleich

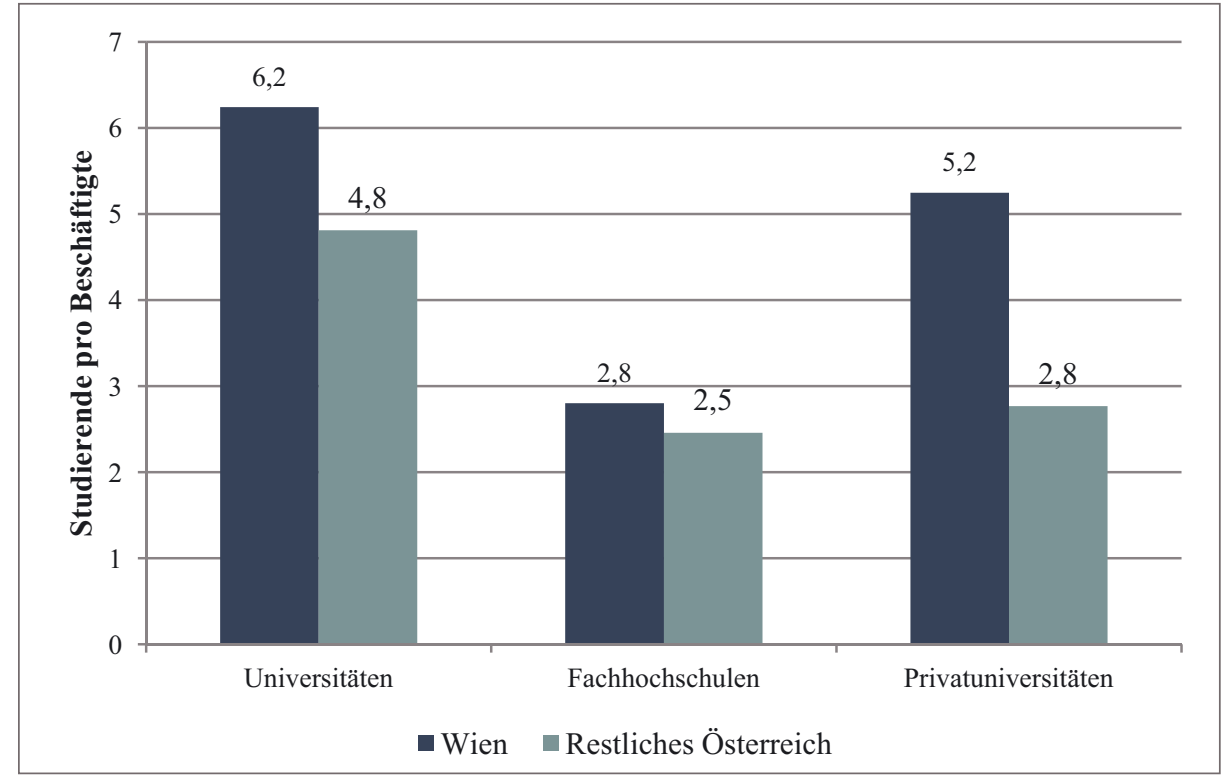

Quellen: uni:data 2013; eigene Berechnungen. 
sondern die gesamten Beschäftigten herangezogen. Um die Vergleichbarkeit zu ermöglichen, wird somit eine gewisse Unschärfe in Kauf genommen. Weiter ist anzumerken, dass sich die Betreuungsverhältnisse zwischen Fakultäten und Studienrichtungen innerhalb der Universitäten ebenfalls stark unterscheiden. Die hier dargestellten Verhältniszahlen erlauben daher nur einen groben Vergleich - hier zwischen Wien und dem restlichen Österreich sowie zwischen den drei Hochschultypen (vgl. Abbildung 4.1). Dabei zeigt sich erstens ein ungünstigeres Betreuungsverhältnis für Einrichtungen aller drei Hochschultypen in Wien gegenüber dem restlichen Österreich, insbesondere bei Privatuniversitäten und den Universitäten. Zweitens sind die Universitäten allgemein vor allem gegenüber den Fachhochschulen im Nachteil; da im universitären Bereich Steuerungsmöglichkeiten wie Studiengebühren oder Studienplatzbeschränkungen - im Gegensatz zu Fachhochschulen und Privatuniversitäten - sehr häufig fehlen.

\subsection{Budget, Finanzierungsstruktur und Drittmittel der Wiener Hochschulen}

Die Finanzierungsstruktur der Wiener Hochschulen unterscheidet sich sehr stark zwischen den Universitäten, die überwiegend vom Bundesministerium für Wissenschaft und Forschung finanziert werden, sowie den Fachhochschulen und Privatuniversitäten, bei denen die öffentliche Hand einen etwas geringeren Finanzierungsanteil ausmacht bzw. andere Gebietskörperschaftsebenen (Land, Gemeinde) eine wichtigere Rolle spielen. Damit unterscheidet sich die Finanzierungsstruktur auch grundlegend

Tabelle 4.3: Finanzierung der Wiener Universitäten durch das Bundesministerium für Wissenschaft und Forschung im Studienjahr 2011

\begin{tabular}{lcccc}
\hline & $\begin{array}{c}\text { Budget 2011 } \\
\text { Mio. Euro }\end{array}$ & $\begin{array}{c}\text { Studienersatzbeitr. Veränderung 2009-2011 (\%) } \\
\text { Budgetanteil } \\
2011(\%)\end{array}$ & Budget & Studierende \\
\hline Universität Wien & 416,338 & 11,2 & 3,9 & 6,5 \\
Medizinische Universität Wien & 353,802 & 1,3 & 5,3 & $-1,4$ \\
Technische Universität Wien & 219,947 & 5,4 & 9,2 & 15,6 \\
Universität für Bodenkultur & 105,429 & 5,1 & 12,9 & 14,9 \\
Veterinärmedizinische Universität & 90,345 & 1,6 & 7,3 & $-3,0$ \\
Wirtschaftsuniversität Wien & 124,841 & 12,0 & 38,0 & $-4,5$ \\
Universität f. angewandte Kunst & 31,400 & 2,7 & 5,5 & 4,2 \\
Univ. f. Musik u. darstellende Kunst & 80,821 & 2,1 & 1,1 & 1,9 \\
Akademie der bildenden Künste & 24,927 & 2,6 & 1,7 & 8,3 \\
\hline Universitäten gesamt & $\mathbf{1 . 4 4 7 , 8 5 0}$ & $\mathbf{6 , 1}$ & $\mathbf{8 , 0}$ & $\mathbf{5 , 9}$ \\
\hline
\end{tabular}

Quelle: BMWF 2013. 
Tabelle 4.4: Finanzierungsstruktur ausgewählter tertiärer Bildungseinrichtungen in Wien

\begin{tabular}{|c|c|c|c|c|}
\hline & \multicolumn{2}{|c|}{ Universität Wien } & \multicolumn{2}{|c|}{ Universität für Bodenkultur } \\
\hline & Anteile $2011(\%)$ & $2009-11(\%)$ & Anteile $2011(\%)$ & 2009-11(\%) \\
\hline Gesamtbudget & 100,0 & 6,9 & 100,0 & 8,3 \\
\hline Wissenschaftsministerium & 79,2 & 6,2 & 71,2 & 11,6 \\
\hline Studienbeiträge & 2,0 & $-39,6$ & 0,8 & $-29,8$ \\
\hline Drittmittel & 13,7 & 22,9 & 25,8 & 3,9 \\
\hline davon: Gemeinde Wien & 0,5 & 43,9 & 0,7 & k. A. \\
\hline davon: EU-Fördermittel & 2,2 & 53,3 & 3,6 & 48,8 \\
\hline davon: FWF & 6,9 & 21,5 & 5,2 & 13,9 \\
\hline davon: Bund (Ministerien) & 1,2 & 9,4 & 3,2 & $-25,6$ \\
\hline \multirow[t]{3}{*}{ Sonstiges } & 5,0 & 13,5 & 2,1 & $-12,9$ \\
\hline & \multicolumn{2}{|c|}{ Veterinärmed. Universität } & \multicolumn{2}{|c|}{ Akademie der bild. Künste } \\
\hline & Anteile $2011(\%)$ & $2009-11(\%)$ & Anteile $2011(\%)$ & $2009-11(\%)$ \\
\hline Gesamtbudget & 100,0 & 14,2 & 100,0 & 4,7 \\
\hline Wissenschaftsministerium & 79,3 & 10,4 & 91,7 & 2,8 \\
\hline Studienbeiträge & 0,2 & $-88,4$ & 0,5 & $-50,5$ \\
\hline Drittmittel & 5,7 & 19,1 & 2,6 & 257,7 \\
\hline davon: Gemeinde Wien & k. A. & k. A. & k. A. & k. A. \\
\hline davon: EU-Fördermittel & 0,9 & 53,8 & k. A. & k. A. \\
\hline davon: FWF & 3,3 & 19,9 & 0,9 & 212,4 \\
\hline davon: Bund (Ministerien) & 1,6 & 5,1 & 0,1 & 130,0 \\
\hline \multirow[t]{3}{*}{ Sonstiges } & 14,8 & 57,8 & 5,2 & 12,4 \\
\hline & \multicolumn{2}{|c|}{ FH Wien } & \multicolumn{2}{|c|}{ FH Campus } \\
\hline & Anteile $2011(\%)$ & 2009-11 (\%) & Anteile $2011(\%)$ & $2009-11(\%)$ \\
\hline Gesamtbudget & 100,0 & 2,5 & 100,0 & 35,0 \\
\hline Wissenschaftsministerium & 78,7 & 1,1 & 58,7 & 36,9 \\
\hline Studienbeiträge & 12,2 & 7,2 & k. A. & k. A. \\
\hline Drittmittel & 4,8 & 22,4 & k. A. & k. A. \\
\hline davon: Gemeinde Wien & 2,9 & k. A. & 6,6 & 49,1 \\
\hline davon: EU-Fördermittel & 0,4 & k. A. & k. A. & k. A. \\
\hline davon: FWF & k. A. & k. A. & k. A. & k. A. \\
\hline davon: Bund (Ministerien) & k. A. & k. A. & k. A. & k. A. \\
\hline Sonstiges & 4,2 & 60,0 & 41,3 & 32,5 \\
\hline
\end{tabular}

Quelle: eigene Erhebung 2013. 


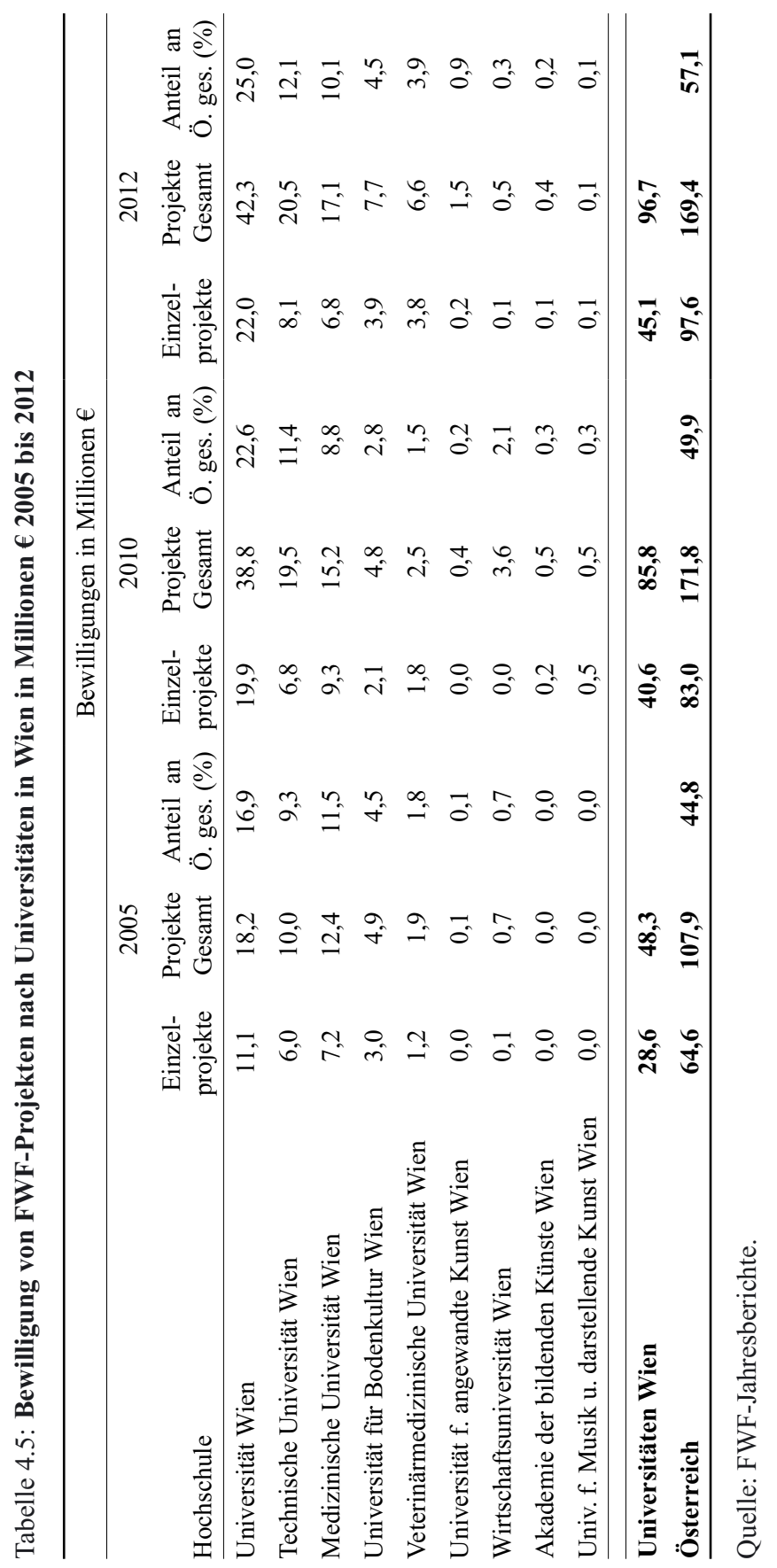


von den Universitäten in Deutschland, die aufgrund des föderalen Hochschulsystems überwiegend von den Ländern finanziert werden. Die neun vom Bundesministerium für Wissenschaft und Forschung finanzierten Universitäten in Wien erhalten ein Budget von rund 1,45 Mrd. Euro (vgl. Tabelle 4.3), wobei der Anteil der Studienersatzbeiträge im Studienjahr 2011 durchschnittlich 6,1 \% ausmacht, jedoch zwischen den einzelnen Universitäten stark schwankt. Auch haben sich in den Jahren 2009 bis 2011 das Budget sowie die Studierendenzahlen sehr unterschiedlich entwickelt (vgl. Wirtschaftsuniversität versus Akademie der bildenden Künste). Wie auch bei den unterschiedlichen Betreuungsquoten spiegelt sich hier einerseits der unterschiedliche Kostenaufwand je Studierenden wieder, sowie die Problematik einer nicht an die Studierendenzahl gekoppelten Finanzierung, wie dies bei Fachhochschulen und Privatuniversitäten sehr wohl der Fall ist. Davon unabhängig ist die Finanzierung durch das Bundesministerium für Wissenschaft und Forschung für die regionale Wertschöpfung der Stadt Wien ein zentraler Faktor.

Wie stellt sich das Gesamtbudget der Wiener Hochschulen dar? Eine Aufschlüsselung ist aufgrund jener Befragung, die im Rahmen dieser Studie durchgeführt wurde, für einen Teil der Einrichtungen möglich (vgl. Tabelle 4.4). Dabei zeigt sich, dass nicht nur für die Universitäten, sondern auch für die Fachhochschulen das Bundesministerium für Wissenschaft und Forschung die zentrale Finanzierungsquelle darstellt. So wird im Fall der FH Wien nahezu ein gleich hoher Budgetanteil durch das Ministerium finanziert wie im Fall der Universität Wien. Studienbeträge spielen seit deren Abschaffung im Jahr 2009 praktisch keine Rolle für die Hochschulfinanzierung.

Umgekehrt nehmen die eingeworbenen Drittmittel eine zunehmend wichtige Bedeutung ein: An der Universität Wien liegt der Beitrag zum Gesamtbudget immerhin bei $13,7 \%$, an der Univ. für Bodenkultur sogar bei $25,8 \%$, während an der Akademie der bildenden Künste sowie der Veterinärmedizinischen Universität die Bedeutung eingeworbener Drittmittel zwar stark steigt, jedoch immer noch vergleichsweise geringe Anteile ausmachen. In Summe steigen in allen untersuchten Fachhochschulen die Drittmittelanteile stark an, was als Ausdruck einer steigenden Wettbewerbsfähigkeit, aber auch eines zunehmenden finanziellen Drucks interpretiert werden kann. Die Wettbewerbsorientierung der Hochschulfinanzierung drückt sich letztlich auch in steigenden Budgets der Förderinstitutionen aus: So vergab der FWF (Fonds zur Förderung wissenschaftlicher Forschung) beispielsweise 2005 107,9 Mio. Euro Fördergelder, 2012 bereits 169,4 Mio. Euro (vgl. Tabelle 4.5). Die Wiener Universitäten konnten an diesen wachsenden Fördervolumina steigende Anteile lukrieren, die 2012 immerhin bei 57,1 \% lagen, was als Ausdruck der Wettbewerbsfähigkeit des Standortes innerhalb der österreichischen Hochschullandschaft zu werten ist.

Allerdings ist die Gewichtung unterschiedlicher Drittmittelgeber zwischen den Hochschulen sehr unterschiedlich: Während im Fall der Universität Wien oder der Universität für Bodenkultur EU-Fördermittel sowie der FWF und die nationale Wissenschaftsförderung an erster Stelle stehen, ist die Gemeinde Wien für die Fachhochschulen der mit Abstand wichtigste Fördergeber. Die gezielten Förderungen der Stadt Wien 
für die Fachhochschulen spielen dabei sicherlich eine zentrale Rolle ${ }^{1}$. Die wichtigste Förderschiene der Stadt Wien ist der WWTF (Wiener Wissenschafts-, Forschungs- und Technologiefonds), dessen Ausschreibungen auf alle Wiener Forschungseinrichtungen abzielen, wobei jedoch der überwiegende Teil der Forschungsgelder an die Universitäten fließt (vgl. Tabelle 4.6). Dabei zeigt sich, dass über die Jahre 2009 bis 2012 über ein Drittel der Fördergelder an die Universität Wien (35,0 \%) geflossen ist, ebenso waren die Technische Universität (26,3\%) sowie die Medizinische Universität (14,9\%) wichtige Empfänger. In der Mittelverteilung der WWTF-Gelder spiegelt sich auch die spezifische Ausrichtung der Calls wider.

Tabelle 4.6: Förderungen des WWTF für Wiener Hochschulen und außeruniversitäre Forschungseinreichteungen (in Mio. Euro)

\begin{tabular}{lccccc}
\hline & 2009 & 2010 & 2011 & 2012 & $\begin{array}{c}2009-2012 \\
(\%)\end{array}$ \\
\hline Universität Wien & 3,9 & 5,5 & 3,5 & 3,8 & 35,0 \\
Technische Universität Wien & 1,7 & 2,9 & 3,6 & 4,3 & 26,3 \\
Medizinische Universität Wien & 1,9 & 0,1 & 3,3 & 1,8 & 14,9 \\
Veterinärmedizinische Universität & 0,4 & 0,8 & 1,1 & - & 4,7 \\
Universität für Bodenkultur & 1,1 & - & 0,2 & - & 2,7 \\
Wirtschaftsuniversität Wien & 0,1 & 0,5 & 0,1 & - & 1,6 \\
Akademie der bildenden Künste & 0,6 & 0,0 & 0,0 & 0,0 & 1,5 \\
Universität für angewandte Kunst & 0,1 & 0,0 & 0,0 & 0,0 & 0,4 \\
Universität für Musik und & - & 0,1 & 0,1 & - & 0,2 \\
darstellende Kunst & & & & & 12,7 \\
Sonstige außeruniversitäre & 1,4 & 2,2 & 1,7 & 0,8 & 100,0 \\
Forschungseinrichtungen & 11,2 & 12,0 & 13,6 & 10,8 & \\
\hline Gesamt & &
\end{tabular}

Quelle: WWTF 2013.

Anmerkung: Inkludiert sind Mittel, die über den WWTF vergeben wurden, d.h WWTF-eigene Mittel sowie die Stadtprogramme „Vienna Research Groups“, „GSK-Impulsprogramm“ und Universitätsinfrastrukturprogramm.

In der aktuellen Förderperiode (2010 bis 2014) unterstützt die Stadt Wien die Wiener Fachhochschulen mit insgesamt 18 Mio. Euro. (Quelle: www.wien.gv.at/forschung/institutionen/ fh.html; 21. August 2013). 